\title{
Bioprocessing of plant-derived virus-like particles of Norwalk virus capsid protein under current Good Manufacture Practice regulations
}

\author{
Huafang Lai ${ }^{a, b}$ and Qiang Chen ${ }^{a, b, *}$ \\ aThe Center of Infectious Diseases and Vaccinology, Biodesign Institute, Arizona State \\ University, Tempe, AZ 85287 \\ ${ }^{\mathrm{b}}$ College of Technology and Innovation, Arizona State University, Mesa, AZ 85212
}

\section{Abstract}

Despite the success in expressing a variety of subunit vaccine proteins in plants and the recent stride in improving vaccine accumulation levels by transient expression systems, there is still no plant-derived vaccine that has been licensed for human use. The lack of commercial success of plant-made vaccines lies in several technical and regulatory barriers that remain to be overcome. These challenges include the lack of scalable downstream processing procedures, the uncertainty of regulatory compliance of production processes, and the lack of demonstration of plant-derived products that meet the required standards of regulatory agencies in identity, purity, potency and safety. In this study, we addressed these remaining challenges and successfully demonstrate the ability of using plants to produce a pharmaceutical grade Norwalk virus (NV) vaccine under current Good Manufacture Practice (cGMP) guidelines at multiple gram scales. Our results demonstrate that an efficient and scalable extraction and purification scheme can established for processing virus-like particles (VLP) of NV capsid protein (NVCP). We successfully operated the upstream and downstream NVCP production processes under cGMP regulations. Furthermore, plant-derived NVCP VLP demonstrates the identity, purity, potency and safety that meet the preset release specifications. This material is being tested in a Phase I human clinical trial. This research provides the first report of producing a plant-derived vaccine at scale under cGMP regulations in an academic setting and an important step for plant-produced vaccines to become a commercial reality.

\section{Keywords}

bioprocessing; cGMP; large-scale; plant-derived vaccines; purification; VLP

\section{Introduction}

Vaccines are one of the greatest life-saving inventions. They have reduced or eradicated many deadly infectious diseases from most of the world and allowed human civilization to flourish in the twenty century. Unfortunately, the availability of these vaccines for use by all those who need them greatly depends on economic considerations such as the cost of their production and delivery. Therefore, a major challenge of vaccine technology is to translate research innovations to economically viable practice. The production of vaccines using plants is a step toward such goal.

"Corresponding author: Qiang Chen, Ph.D. Arizona State University, Mail Zone 5401, 1001 S. McAllister Avenue, Tempe, AZ 85287. T: (480) 239-7802, F: (480) 727-7615. qiang.chen.4@asu.edu. 
Currently licensed subunit vaccines are produced in mammalian or microbial cell cultures which require large capital investment for facility construction and the subsequent high-cost operations (Thiel 2004). Therefore, the cost for producing vaccines in these production systems may be too prohibitive for resource poor health care systems, and may prevent the full realization of the vast health-benefit potential of vaccines. As such, the development of vaccine production platforms that are cost-effective, scalable, and safe is urgently needed.

Plants offer a viable alternative system for subunit vaccine production due to their ability to produce large amounts of recombinant protein at low cost, their eukaryotic protein processing ability to assemble and modify recombinant proteins properly, and their the lowrisk introduction of adventitious human or animal pathogens (Chen 2011; Faye and Gomord 2010). In contrast to cell culture-based production systems, plant biomass generation does not require the construction of expensive fermentation facilities, and correspondingly, scaleup production can be achieved without the need to construct duplicate facilities. As a result, plant biomass generation and upstream processing capacity can be operated and scaled in a capital-efficient manner with established agriculture practice. This flexibility and costsaving benefit cannot be easily matched by current fermentation-based technologies. Therefore, plants offer unique advantages for the production of subunit vaccines in terms of scale, speed, costs, yield, and safety. As a result of these advantages, diverse subunit vaccines have been expressed in a variety of expression vectors and host plant species (Chen 2011; Rybicki 2010). The results from these expression studies collectively demonstrated that plants are capable of expressing a broad portfolio of diverse subunit vaccines and that some of these plant-derived antigens are able to induce active protective humoral and cellmediated immune responses (Paul and Ma 2010; Rybicki 2010). Despite this progress, there is still no licensed plant-derived vaccine on the market for human use. The initial challenge of producing subunit vaccines in plants was the slowness and low and inconsistent yield of antigens in transgenic plants. This problem reflects the inherent limitations of the early expression system using stable transgenic plants: the lack of strong regulatory elements to drive target protein accumulation and the position effect caused by the randomness of transgene integration in plant genome (Chen 2008; Davies 2010). The low production yield of these early plant expression systems made vaccine production impractical and greatly reduced the cost-saving benefit of plant expression system (Chen, et al. 2009). The development of plant-virus based transient plant expression systems has overcome the challenges of vaccine production speed and yield (Komarova, et al. 2010; Lico, et al. 2008). As reported by our group and others, high levels of subunit vaccines can be produced in tobacco plant leaves within 1-2 weeks of vector infiltration with a tobacco mosaic virus (TMV) RNA replicon (the MagnIcon) system or a geminiviral DNA replicon system based on bean yellow dwarf virus (He, et al. 2011; Huang, et al. 2009; Lai, et al. 2011; Santi, et al. 2008). There are, however, several remaining challenges that must be overcome before plants can become a production platform for licensed subunit vaccines. They include issues associated with the scalability of downstream processing, regulatory compliance, and the quality equivalency of plant-derived vaccines with those produced by traditional cell-based systems.

Norwalk viruses (NV) are a group of highly infectious viruses that are responsible for $>95 \%$ of epidemic viral gastroenteritis in adult humans (Patel, et al. 2008). The NV capsid is an icosahedral $38 \mathrm{~nm}$ structure assembled from 90 dimers of the $58 \mathrm{kDa}$ capsid protein $(\mathrm{CP})$ arranged in a T=3 symmetry (Prasad, et al. 1999). Expression of recombinant NV capsid proteins (NVCP) in insect cells resulted in the formation of self-assemble empty virus-like particles (VLP) that resemble authentic virus particles (Jiang, et al. 1992). The VLP of $\mathrm{NVCP}$ is a major candidate for vaccine development of NV, because it is highly immunogenic and efficiently has been shown to trigger both B- and T-cell responses (Jennings and Bachmann 2007). In addition, the repetitive VLP surface structure and its 
particulate form promote activation of B-cells and binding of antibodies, thus enhancing uptake by antigen-presenting cells (APC). NVLP VLP can be delivered orally or intranasally and can induce anti-NVCP secretory IgA at distal mucosal sites, providing further support for vaccine development (Ball, et al. 1998). There is also substantial interest in using NVCP VLP as a platform to display heterologous peptide epitopes to take advantage of the immunogenicity-enhancing features of VLP (Herbst-Kralovetz, et al. 2010). Our previous studies demonstrated that NVCP VLP can be rapidly produced at high levels in plants by using transient expression systems based on tobamovirus (Santi, et al. 2008) and geminivirus (Huang, et al. 2009; Lai, et al. 2011) vectors. Our studies also demonstrated that the plant-derived VLP appear to be very similar both morphologically and antigenically to insect cell-derived particles (Lai, et al. 2011; Santi, et al. 2008).

For the promise of plants as an alternative for vaccine production to become a reality, the technology has to approve its ability to produce subunit vaccines at a relevant scale and with product qualities (identity, purity, potency and safety) that meet the required standards of regulatory agencies such as US Food and Drug Administration (FDA). In this study, we aim to use NVCP VLP as a model vaccine to address these issues and to demonstrate the ability of using plants to produce pharmaceutical grade vaccines under federal current Good Manufacturing Practices (cGMP) guidelines at multiple gram scales. To reach these goals, we established and characterized Agrobacterium tumefaciens and Nicotiana benthamiana master and working banks for NVCP production, optimized conditions for large-scale plant growth and infiltration, developed scalable and cGMP compliant procedures for NVCP VLP extraction and purification, and identified and developed product analytical assays. Plantderived NVCP VLP produced by this process is highly pure and retained VLP structure and potent immunogenicity in mice. This research provides the first case study report of producing a plant-derived vaccine at scale under cGMP regulations in an academic setting.

\section{Materials and Methods}

\section{Expression vectors and sequence confirmation}

The construction of MagnICON vectors for NVCP (pICH3NVCP, pICH15879, and pICH14011) and the green fluorescent protein (GFP) (pICHGFP, pICH15879, and pICH14011) expression has been extensively described previously (Giritch, et al. 2006; Santi, et al. 2008). No new expression vector was constructed for this research. The identity and sequence of these expression vectors in the A. tumefaciens master and working banks were confirmed by DNA sequence analysis of both strands with an Applied Biosystems 3730 capillary sequencer following the manufacture's instruction (Applied Biosystems).

\section{N. benthamiana plant biomass generation}

A master seed bank of wild-type was obtained from Kentucky Bioprocessing, LLC (KBP). A working seed bank was established from the master seed bank. Two seeds from the working seed bank were sowed to one Jiffy-7 peat pellet (Hummert International). One hundred and twenty (120) peat pellets were incubated in water in a 210-square inch growth tray covered with a humidity dome (Hummert International). The humidity dome was removed one week later and plants were grown in a greenhouse at $25 \pm 0.5^{\circ} \mathrm{C}$ with a $16 \mathrm{hr}$ Light $/ 8 \mathrm{hr}$ dark cycle of $85 \%$ white spectrum light supplemented with blue spectrum light for 4 more weeks. After the first week, plants were irrigated with a Jack's all purpose fertilizer (10-10-10) at a concentration of $1.5 \mathrm{~g} / \mathrm{L}$. For a comparative study, plants were also grown under natural light with other conditions remained the same as plants grown under artificial light. Seed germination rate were calculated by counting the number of seedlings in 10 growth trays (600 peat pellets) and then dividing the seedling number by the number of 
seeds that were sowed in those peat pellets (1,200 seeds). At various stages of plant growth, leaves from 10 individual plants were harvested and weighted to calculate the biomass yield.

\section{Establishment of A. tumefaciens cell banks}

Plant expression vectors were individually transformed into A. tumefaciensstrain GV3101 by electroporation and cultured as previously described (Santi, et al. 2008). GV3101 cells from a single colony harboring each exoressuib vector were grown at $28 \pm 0.5^{\circ} \mathrm{C}$ until $\mathrm{OD}_{600}=$ $1.0 \pm 0.2$. These cells were aliquoted $(1 \mathrm{ml})$ and preserved in multiple vials with $20 \%$ glycerol at $-80 \pm 4{ }^{\circ} \mathrm{C}$ as the master cell bank. A working cell bank (100 ul per vial) for each master bank was established with a similar procedure.

\section{Agroinfiltration of $\boldsymbol{N}$. benthamiana plants}

A. tumefaciens GV3101 cultures for the three vector modules of the MagnICON system were started by inoculating cells from the corresponding cell working bank. The GV3101 cultures were incubated until the $\mathrm{OD}_{600}$ reached $1.0 \pm 0.2$ as we previously described (Huang, et al. 2010; Lai, et al. 2010; Santi, et al. 2008). GV3101 cells were then harvested by centrifugation and resuspended in Agroinfiltration media (Santi, et al. 2008). The three GV3101 cultures were then combined in a 1:1:1 ratio to yield a total $\mathrm{OD}_{600}$ of $0.20 \pm 0.02$. This final infiltration GV3101 culture mixture was transferred into a reservoir in the infiltration container. 5-week old plants were inverted by $180^{\circ}$, loaded on a rack, and transferred into the infiltration container in which the plants were submerged into the GV3101 liquid mixture. Co-Agroinfiltration of plants with GV3101 strains containing the 3' (pICH3NVCP or pICHGFP), 5' (pICH15879) and the integrase (pICH14011) MagnICON modules were achieved by applying and releasing a vacuum $(2 \times 2$ minutes, $100 \mathrm{mbar})$ to allow entrance of GV3101 solution into the interstitial spaces of submerged plant tissues. Since plants were grown in peat pellets covered with nets, there was no need to cover growth containers to prevent contamination of infiltration solution by loose soil debris. After infiltration, plants were inverted back to the upright position and transferred back to the growth room for NVCP or GFP expression.

\section{Extraction of total protein from plant leaves}

Agroinfiltrated $N$. benthamiana leaves were harvested on days 6,7, and 8 days post infiltration (dpi) for evaluating the temporal pattern of pHu-E16 MAb expression. For other protein analysis or NVCP purification, plant leaves were harvested $7 \mathrm{dpi}$. Total leaf protein was extracted by homogenization with extraction buffer $(25 \mathrm{mM}$ sodium phosphate, 100 $\mathrm{mM} \mathrm{NaCl}, 50 \mathrm{mM}$ sodium ascorbate, $2 \mathrm{mM}$ PMSF, $\mathrm{pH}$ 5.75) with a blender following the manufacture's instruction. The crude plant extract was clarified by centrifugation at $14,000 \times$ $g$ for $10 \mathrm{~min}$ at $4^{\circ} \mathrm{C}$.

\section{NVCP VLP extraction and purification}

The downstream processing of NVCP VLP production is summarized in Fig. 4. $N$. benthamiana leaves were harvested $7 \mathrm{dpi}$ and homogenized by blending in extraction buffer at a ratio of $1 \mathrm{~L}$ of extraction buffer per Kg leaf fresh weight (LFW). Plant-specific protease inhibitor tablet (Sigma, 5 tablets per L of buffer) was included in the extraction buffer during process development, but was found unnecessary. After 1 hour of incubation at $4{ }^{\circ} \mathrm{C}$, homogenates were centrifuged at $6000 \times g$ for 30 minutes at $4{ }^{\circ} \mathrm{C}$ to remove leaf debris and the plant photosynthetic enzyme ribulose 1,5-bisphosphate carboxylase-oxygenase (RuBisco) precipitated at the low $\mathrm{pH}$ condition. The supernatant was then incubated at $4{ }^{\circ} \mathrm{C}$ for 24 hours and centrifuged for 30 minutes at $6000 \times g$ at $4{ }^{\circ} \mathrm{C}$ to further remove RuBisco precipitated during incubation. This process was repeated totally 3 times to future remove the residual RuBisco. The supernatant was then filtered with a $0.22 \mu \mathrm{M}$ filter (Millipore). 
The clarified supernatant was then concentrated by ultrafiltration/diafiltration (UF/DF) with a 100kDa polyethersulfone tangential flow (PES TFF) membrane (Pall Corporation). The UF/DF process was also aimed to remove any residual contaminating small molecule. The UF/DF retentate was then subjected to an anion exchange (IEX) chromatography with diethylaminoethyl cellulose (DEAE) Sepharose FF resin (GE Healthcare) with a linear velocity of $60 \mathrm{~cm} / \mathrm{hr}$. A final UF/DF step (100 kDa, PES TFF membrane) was used to concentrate the purified product in the formulation buffer $(25 \mathrm{mM}$ sodium phosphate, 100 $\mathrm{mM} \mathrm{NaCl}, \mathrm{pH}$ 5.75) and to remove any remaining small molecules prior to sterile fill in a class 100 Biosafety hood. The purity of NVCP VLP and the level of residual DNA in the final purified sample were determined using a densitometer and a commercial PicoGreen dsDNA quantitation kit (Invitrogen), respectively as described preciously (Lai, et al. 2010; Lai, et al. 2011). The concentration of purified NVCP VLP was measured by measuring the $\mathrm{OD}$ at $280 \mathrm{~nm}$ (A280) with extinction coefficient of 0.852 as described previously (Ausar, et al. 2006).

\section{SDS-PAGE and Western blot}

In-process or purified protein samples were subjected to 4-20\% gradient SDS-PAGE under a reducing (5\% v/v $\beta$-mercaptoethanol) condition. Gels were either stained with Coomassie blue, silver or used to transfer proteins onto polyvinlidene difluoride (PVDF) membranes (GE Healthcare) as described previously (Santi, et al. 2008). A rabbit anti-NVCP polyclonal antibody (Santi, et al. 2008) and a horseradish peroxidase (HRP)-conjugated goat antirabbit-IgG (Sigma) were used in a 1:10,000 dilution as detection antibodies for western blot analysis. A insect cell-derived NVCP VLP (iNVCP) (Jiang, et al. 1992) was used as reference standard. The membranes were developed with were developed with 3,3',5,5'tetramethylbenzidine (TMB) substrate (KPL Inc.)

\section{ELISA}

NVCP quantitative ELISA was performed to quantify NVCP expression in plants as previously described (Santi, et al. 2008). Briefly, plates were coated with a rabbit antiNVCP polyclonal antibody. After incubation with plant protein extract or purified NVCP, a guinea pig anti-NVCP serum and a HRP-conjugated goat anti-guinea pig IgG (Santi, et al. 2008) were used as the detection antibodies. iNVCP VLP was used as reference standard. The plates were developed with TMB substrate (KPL Inc).

\section{$\mathrm{N}$-terminal sequencing, sucrose gradient, electron microscopy and GFP visualization}

$\mathrm{N}$-terminal sequencing of NVCP, sucrose gradient centrifugation and electron microscopy for NVCP VLP, and GFP visualization were all performed according to published protocols (Huang, et al. 2009; Santi, et al. 2008; Speicher, et al. 2001).

\section{Results}

\section{Establishment of facilities for cGMP production of NVCP VLP from plants}

We have established greenhouse and bioprocessing facilities for cGMP production of NVCP VLP (Fig. 1). The new and purposefully-built production facilities permit bioprocessing of NVCP vaccines and other plant-made pharmaceuticals (PMPs) under cGMP Quality Management System (QMS). As shown in Fig. 1c, the $2000 \mathrm{ft}^{2}$ central bioprocessing suite has separate rooms for buffer preparation, incoming samples, chromatography and sterile fill. The facility has differential pressure and the final chromatography room contains a Class 100 environment for finishing and vial fill. The facility was designed to have separated unidirectional flow of human workers and biological material, so that the product will meet the requirements of both United States (US) and European Union (EU) regulatory 
agencies for manufacturing of human pharmaceuticals. The central bioprocessing suite is accompanied by a quality control (QC)/Quality Assurance (QA) laboratory for product quality management and a laboratory for process development (Fig. 1c). Greenhouses facilities are an integral part of the production facility. We established a $3600 \mathrm{ft}^{2}$ Biosafety level 2 (BSL-2) greenhouse facility that provides plant production space, a potting room, autoclave, an acclimation room, and is coordinated by fully integrated computer control for climatic control and monitoring (Fig. 1a). The BL-2 greenhouse is equipped with advanced technology for containment of plants or pathogens. The facility design features fully sealed walls and ceilings, screened air intake and exhaust ports, non-porous concrete floor, centralized floor drainage system, tight sealing doors and walls that were meticulously inspected and sealed with caulking. This level of greenhouse containment is necessary to minimize the risk of inadvertent genetic contamination of the external environment, and to maintain the integrity of the plant-derived vaccines. This greenhouse is equipped with a computer-monitored climate-control system through the central control panel, manufactured by Argus Environmental Control Systems. Each of four greenhouse cells has bench-top heating system. All heating, cooling, and ventilation of the greenhouses are constantly monitored by the computerized control systems, and adjustments made as needed throughout the day. The established bioprocessing facilities and QA/QC laboratory have been certified for producing human vaccines.

\section{Establishment of $\boldsymbol{A}$. tumefaciens and $\boldsymbol{N}$. benthamiana banks for NVCP production}

To generate plant material for cGMP-compliant production of NVCP, master and working banks of A. tumefaciens strains that harboring the NVCP expression vectors and a seed bank of wild-type $N$. benthamiana for growing biomass for transient transformation must be established and qualified by the QMS. Three A. tumefaciens master banks, each harboring the optimal DNA construct for NVCP transient expression in tobacco mosaic virus (TMV) 3' module (pICH3NVCP), TMV 5' module (pICH15879), and integrase (pICH14011) of the MagnICON system (Giritch, et al. 2006) have been established in the bacterial strain of GV3101. Each master bank contains 5000 vials of $1 \mathrm{ml}$ exponential phase GV3101 culture and has been stored at $-80 \pm 4^{\circ} \mathrm{C}$. The identity of the A. tumefaciens host strain and functional genes and vectors related to NVCP expression are fully described and sequenced. Specifically, the sequencing of the pI3NVCP, pICH15879, and pICH14011 plasmid has been performed and yielded the expected correct DNA sequence for each plasmid. Sequencing analysis was performed for both strands of DNA and in each direction for at least 2 reads. The genetic stability of these banks has been repeatedly characterized by DNA sequencing and product identity tests (NVCP western and ELISA, Fig.3 and Table 3) over a four-year period. Our results indicate that the strain in each GV3101 bank retains its genetic identity and consistently produces the indented NVCP VLP product after $>20$ passages and $>100$ generation time in liquid culture, and after 4 years of storage at $-80 \pm 4{ }^{\circ} \mathrm{C}$. Analysis of corresponding GV3101 working banks yielded similar identity and stability results. The results of genetic and product identity tests of the "end-of production" GV3101 cells over 10 production runs also have confirmed the stability of these banks. A master seed bank for wild-type $N$. benthamiana is also needed to produce plant material for NVCP transient expression. We have obtained such documented $N$. benthamiana seed bank from KBP. This seed bank was characterized for several parameters including germination rate, seedling phenotype, and growth rate. Our results showed that $N$. benthamiana seeds in the bank have a germination rate of $99 \%$ and produce uniform seedlings with typical $N$. benthamiana morphology (Fig. 2). Similar results were obtained with seeds that had been stored at $4 \pm 2{ }^{\circ} \mathrm{C}$ for 4 years. Results of these characterizations collectively demonstrated that master and working banks of $A$. tumefaciens strains and $N$. benthamiana seeds have meet the qualification standards of identity and stability, and can be used for cGMP production of NVCP. 


\section{Scale-up plant growth and infiltration}

To produce the maximal amount of leaf biomass and target recombinant protein per square meter of green house space, we investigated multiple factors that affect plant growth and Agrobacterium infiltration. Our results indicated that both plant health and development stage determine the speed at which MagnICON systems (Giritch, et al. 2006) drive protein expression and the level to which NVCP will accumulate. Specific parameters which have impacts on biomass generation and protein expression levels include greenhouse temperature, light source and intensity, plant inoculation age, and time required for the maximum accumulation of target protein after leaf infiltration. We first compared plant growth and NVCP productivity under natural and artificial light. As shown in Fig. 3a, while plants grown under nature light yielded more leaf biomass, the NVCP yield per LFW is much less than that grown under artificial light and fluctuates widely between batches. In addition, the dark green leaves from natural-light grown plants yielded more solid cell debris (data not shown) that complicated the downstream processing of NVCP. Therefore, using artificial light is the method of choice for biomass generation for NVCP production to get higher and consistent product yield. Results of light cycle and temperature experiments (data not shown) indicate that a $16 \mathrm{hr} \mathrm{Light/} / 8 \mathrm{hr}$ dark cycle at $25 \pm 0.5^{\circ} \mathrm{C}$ is the optimal condition to generate biomass under such artificial lighting. Our results also indicate that under these conditions, 5-week plants (Fig. 2d) provide the optimal material for NVCP production, because it accumulates the highest level of NVCP (Fig. 3b, red column) while the biomass yield is sufficiently high. Plants older than 5-weeks produce more biomass (Fig. 3b, green square), but are too tall for the efficient use of growth and infiltration chamber space. Furthermore, flower development was observed for $N$. benthamiana plants after 5 weeks of growth (data not shown). As a result, using biomass post 5-week stage would increase the risk of introducing extra alkaloids and phenolics into the purification feeds stream. Thus, the 5-week growth period provides the best balance point for the combined need of biomass generation, NVCP accumulation, space efficiency, and the ease of filtration operation. We also investigated the space requirement for generating sufficient cGMP grade NVCP VLP for Phase I human clinical trial. Our results indicate that growing $N$. benthamiana in Jiffy-7 peat pellets (Fig. 2) uses only $1 / 10^{\text {th }}$ of the space that is required for using 4 -inch pots. Since growth media in peat pellets are covered by a mesh (Fig. 2a), the use of peat pellets eliminates the need to cover loose soil, a common practice required for using regular growth pot before inverting plants for infiltration. Thus, using peat pellets reduces the time and overall cost of NVCP production. Collectively, these results allowed us to establish the following conditions for the optimal plant growth and NVCP expression. One hundred twenty (120) $N$. benthamiana plants were planted and grown in 60 Jiffy-7 peat pellets in a 210 square inch ( 0.14 square meter) tray for 5 weeks in a greenhouse at $25 \pm 0.5^{\circ} \mathrm{C}$ with a $16 \mathrm{hr}$ Light/8hr dark cycle of $85 \%$ white spectrum light supplemented with blue spectrum light. Under such conditions, each tray produces $250 \pm 5 \mathrm{~g}$ of green biomass. Therefore, biomass for producing sufficient amount of purified NVCP for a Phase I clinical trial can be generated by 40 such production trays in 5 weeks. This is equivalent to 5.6 square meters of green house space. Since we stack 3 growth trays vertically in light racks, the required horizontal greenhouse space was reduced to 1.87 square meters. The horizontal space requirement could be further reduced by stacking more layers of growth trays vertically.

To efficiently introduce Agrobacterium into plants, we have developed Standard Operating Procedures (SOPs) for growing Agrobacterium culture and vacuum infiltration of $N$. benthamiana plants at the scale needed for producing the Phase I clinical trial material. These SOPs include specific parameters for the optimal introduction of Agrobacterium into plant cells such as culture time, media condition, culture $\mathrm{OD}_{600}$, infiltration vacuum pressure, and infiltration time. To facilitate large-scale operations, we also designed a vacuum tunnel to accommodate 16 trays of plants per infiltration cycle with automation. Our 
results demonstrated that plants infiltrated based on the SOPs allow efficient infiltration of vector-carrying GV3101 Agrobacteria in the entire leaf area as demonstrated by the expression of the GFP (Fig. 3c). ELISA analysis indicated that $N$. benthamiana plants grown and infiltrated under above scale-up conditions reached the highest level $(\sim 400 \mathrm{mg} / \mathrm{kg}$ LFW, Figure 3a and b) of NVCP protein in leaves 7 dpi. Thus, the temporal expression pattern, as well as the level of NVCP accumulation under these scale-up conditions, are similar to results of our previous NVCP experiments in smaller bench-scales (Santi, et al. 2008), supporting the scalability of our upstream process.

\section{NVCP VLP production, extraction and purification}

Traditional NVCP extraction and purification methods based on ultracentrifugation (Jiang, et al. 1992; Prasad, et al. 2000) cannot be realistically scale-up for production of this vaccine at manufacture scale. As a result, extraction and purification process development was performed to optimize the procedure for scale-up production of plant-derived NVCP under cGMP regulations. To comply with cGMP regulations, buffers and solutions were prepared with Water For Injection (WFI) water (Hyclone). New chemicals, reagents, and disposable materials were selected and dedicated to use only for this project. Dedicated reusable materials such as columns, resins, and filtration cassettes were sanitized with $0.1 \mathrm{~N} \mathrm{NaOH}$ for 30 minutes before and after each cycle of use. The process development work allowed us to establish a qualified production process for NVCP (summarized in Fig. 4). Based on this process, NVCP VLP was extracted and purified by a three-step process comprised of low $\mathrm{pH}$ precipitation, UF/DF with a TFF membrane, and DEAE-anion exchange chromatography. Our results showed that precipitation at $\mathrm{pH} 5.75$ effectively removed the most abundant plant host protein, the photosynthetic enzyme RuBisCo, and other plant proteins (Fig. 5, Lane 4). DEAE anion-exchange chromatography removed the remaining contaminating proteins and enriched NVCP to greater than $95 \%$ purity (Fig. 5, Lane 5). To validate the scalability of our processing protocol, we performed NVCP processing using different scales of plant materials ranging from 100 to 15,000 grams. The results indicate that our process is highly scalable and can produce NVCP with consistent high purity and recovery among batches of different scales (Table 1). In total, $>4 \mathrm{~g}$ of purified NVCP VLP was produced under cGMP regulations. The production, extraction and purification process established from this study was transferred to KBP along with Agrobacterium stains from master banks for future manufacture-scale production of NVCP VLP. Part of the purified NVCP VLP was also delivered to KBP for final vial fill and releasing for clinical testing.

\section{Identification and development of product analytical assays and characterization of plant- derived NVCP VLP}

To ensure the quality of the final product and in-process samples, we have identified and developed assays essential for the cGMP compliance (Table 2). These assays were designed to monitor the in-process samples and to ensure that the final product meets preset specifications for the release of human pharmaceuticals in identity, purity, concentration, tertiary structure, functionality, and in concentrations of host contaminating molecules. Our results showed that the identity of plant-derived NVCP was confirmed by both western blot and $\mathrm{N}$-terminal sequencing analysis (Table 3 ). Electron microscopy and sucrose gradient centrifugation analysis also confirmed that NVCP formed the typical 38nm VLP indistinguishable from insect-derived VLP, as we reported previously (Huang, et al. 2009; Lai, et al. 2011; Santi, et al. 2008). The final product is $>95 \%$ pure at a concentration of $10 \pm 0.3 \mathrm{mg} / \mathrm{ml}$ and was colorless and particle-free in appearance (Table 3). The level of residual DNA in the final NVCP product was found below the FDA specification for injectable human pharmaceuticals (Table 3). 
The potency of the purified NVCP VLP was demonstrated by its ability in inducing strong $\mathrm{NVCP}$-specific systemic, local and distal mucosal immune responses in mice following intranasal administration (Velasquez, et al. 2011) The final product are being further analyzed at KBP. Overall, results from current analysis indicate that NVCP VLP produced from this scale-up process meets the predetermined release specifications in identity, purity and potency.

\section{Discussion}

Despite the tremendous progress in the research and development of plant-made vaccines, currently there is still no licensed plant-derived vaccine for human use. The history and current status of plant-made NVCP as a vaccine candidate clearly illustrates the progress and remaining challenges in the PMP field in general. The first attempt of expressing NVCP in plants was performed with stable transgenic plants (Zhang, et al. 2006). As for other early PMPs expressed in transgenic plants, it took several months to a year to generate and select NVCP-expressing plant lines and the yield of NVCP was very low $(\sim 10-20 \mu \mathrm{g} / \mathrm{g}$ tissue fresh weight). We have successfully resolved these issues associated with stable transgenic plants by using transient expression systems based on plant virus replicons. For example, our previous reports indicate that these transient expression systems allow us to achieve highlevel NVCP accumulation ( $\sim 400 \mu \mathrm{g} / \mathrm{g}$ LFW) within 1-2 weeks of vector infiltration. However, like for other PMPs, there are remaining technical and regulatory hurdles that must be overcome before plant-derived NVCP can be accepted as a licensed vaccine product. These challenges include the development of scalable downstream processing procedures to recover NVCP VLP plant leaves, the compliance of FDA cGMP regulation or the operation, and ultimately the demonstration of the identity, purity, potency and safety of plant-made NVCP VLP vaccine that meet the required standards of regulatory agencies. In this study, we tackled these remaining challenges and successfully demonstrated the ability of using plants to produce pharmaceutical grade vaccines under cGMP guidelines at multiple gram scales. Our results specifically show that (1) an efficient, scalable purification process was established for NVCP VLP to address the scalability issue (2) we successfully operated the upstream and downstream NVCP production process under cGMP guidelines, and (3) The identity, purity, potency and safety of plant-derived NVCP VLP produced at scale meet the preset release specifications. This material is being tested in a Phase I human clinical trial. This research provides the first report of producing a plant-derived vaccine at scale under cGMP regulations in an academic setting and an important step for PMPs to become a commercial reality.

Downstream processing is an important component of pharmaceutical protein production. As expression NVCP levels increase, purification costs become an increasingly significant proportion (>80\%) of the total cost of NVCP and other vaccine production (Chen 2011; Chen 2011; Faye and Gomord 2010; Rybicki 2010). Thus, the development of methods that can efficiently purify NVCP from plant tissue at large-scale is essential for plant expression system to be considered a viable production platform candidate and for the full realization of economic effectiveness of PMP technology.

Traditionally, NVLP and other VLP have been recovered based only on a few variations of centrifugation and precipitation methods (Jiang, et al. 1992; Prasad, et al. 2000; Santi, et al. 2008). VLP are routinely purified on the basis of their size and density using gradient ultracentrifugation techniques with sucrose and cesium chloride (CsCl) (Estes 2004). Ultracentrifugation and density gradient methods are effective isolation tools for isolating bench-scale quantities of NVLP for research. However, they are not practical for large-scale commercial vaccine manufacture because they are difficult to scale up, time-consuming, and produce poor yields (Rolland, et al. 2001). The unique properties of plant tissues also require 
specific considerations for purification method development. For example, plants produce more solid debris, therefore, direct loading of plant extracts onto chromatographic columns often causes resin fouling and poor binding of NVCP to the resin (Chen 2008). The carbon assimilation enzyme RuBisCo is the major contaminating protein in plant leaves, therefore, should be removed from the extract prior to the chromatographic steps.

This research has allowed us to successfully develop an extraction and purification process that effectively circumvents the scale limitations of traditional VLP purification methods and allow efficiently purification of NVCP from $N$. benthamiana leaves. Our results show that the low-pH precipitation step effectively eliminates plant solid debris and RuBisCo from the downstream processing feed stream, therefore, effectively resolves the specifically challenges presented by the plant tissue. We also explored column chromatography for its ability to achieve high purity and recovery rates, and its facile adaptability for scaling up and cGMP manufacturing (Chen 2011). Our results showed that DEAE anion-exchange chromatography is an optimal method for the scalable purification of NVCP VLP. Collectively, we demonstrated that our downstream process that bases on low $\mathrm{pH}$ precipitation and a DEAE chromatographic step is highly scalable, and can produce fully assembled VLP with high product purity ( $>95 \%)$, yet eliminates the laborious and timeconsuming steps of sucrose and $\mathrm{CsCl}$ gradients. This newly-developed downstream process will increase manufacturing productivity, reduce cost of operations, enhance scalability for processing large volumes of NVLP preparations with high yield, and preserve the stability of the VLP. Since precipitation and DEAE chromatography have been successfully employed for cGMP production of licensed pharmaceutical products, this ensures the compliance of our manufacturing procedures with the FDA's cGMP regulations. SOPs based on this processing scheme have been established and used in cGMP production of NVLP for a Phase I human clinical trial.

The demonstration of regulatory compliance of the production process and product quality is another remaining challenge presented to the PMP field (Chen 2011; Faye and Gomord 2010). This critical issue has been difficult to resolve due to the lack of interest from pharmaceutical industry for "unproven" technology and the unusual high financial demand for academia. As the first step, we have successfully established cGMP-compliant greenhouses, a central bioprocessing suite, and a QA/QC facility. The design and construction of these facilities have been certified in full compliance of cGMP regulations for pharmaceutical products of both US and EU markets. The establishment of these dedicated facilities laid the foundation for producing NVCP VLP vaccine that can be use for human clinical trials and its future commercial application. We next established three $A$. tumefaciens master stock cell banks, and an $N$ benthamiana seed bank and their corresponding working banks for providing cGMP upstream materials. These banks have been extensively characterized for their identity and stability, and they have been shown to meet the required specifications. We also established the optimal conditions for large-scale biomass generation and infiltration with A. tumefaciens strains that are carrying the NVCP expression vectors. As we discussed earlier, the downstream processing procedure developed from this study is not only effective and scalable, but also uses materials and techniques that are routinely used for the production of licensed pharmaceutical products, therefore, is fully in compliance with cGMP regulations. Our study also demonstrated the low-cost nature of our plant-based production system. For example, the material cost for generating the plant biomass for one gram of NVCP is approximately $\$ 15$ at our production scale. In contrast, the material cost (protein-free culture media and a disposable bioreactor) would be at least $\$ 390$ if the biomass for the same amount of NVCP were generated in a mammalian cell culture. Furthermore, the capital investment required for building our greenhouses is significant less than that of an equivalent mammalian cell culture fermentation facility (Elbehri 2005). 
In addition to show the cGMP-compliance of the production process, we also aim to demonstrate that NVCP VLP produced by this process exhibits qualities that meet the requirements of regulatory agencies. First, we identified and established analytical assays for in-process samples and the final product. Results indicate that the identity and structure of plant-derived NVCP VLP is indistinguishable from the insect-cell derived NVCP VLP standard. The purity of the final purified NVCP VLP is $>95 \%$ and was shown to effectively induce potent NV specific immune responses in mice (Velasquez, et al. 2011). Therefore, the purity, potency and other characteristics also meet the preset release specifications for Phase I human clinical trials. As part of the QMS, we have established a documentation system that documents all aspects of NVCP production process. The dossier includes SOPs for facility validation, bacterial and seed bank characterizations, operations for upstream and downstream processing, and QA/QC analytical procedures.

In summary, we demonstrate that a vaccine candidate based on NVCP VLP can be successfully produced in plants at scale and in compliance of FDA's cGMP regulations. The plant-derived NVCP VLP demonstrated the quality that meets the preset release specifications and is being tested in a Phase I human clinical trial. Such demonstration has brought us one step closer to overcome the remaining challenges of the PMP field, and illustrated the feasibility of using plants as a cost-effective and scalable production platform for commercial vaccines. The establishment of cGMP-compliant PMP production facilities and a documentation dossier in an academic setting will facilitate translational research for other PMP products.

\section{Acknowledgments}

The authors wish to acknowledge Dr. Charles Arntzen for his generous support and Dr. Yuri Gleba (Icon Genetics, Germany) for the MagnICON system. We thank Gary Morris, Bingying Jiang, Linh Nguyen, Julie Featherston, Kadie Gavan, Darin Stone, and Yihuang Lu for contributing to biomass generation, protein processing and product technical analysis. We also thank KBP for the seed bank of $N$. benthamiana. This work was supported by a startup fund from the Biodesign Institute of Arizona State University to Q. C. and in part by the National Institute of Allergy and Infectious Diseases grant \# U19 AI 062150-Production Core.

\section{References}

Ausar SF, Foubert TR, Hudson MH, Vedvick TS, Middaugh CR. Conformational stability and disassembly of norwalk virus like particles: Effect of $\mathrm{pH}$ and temperature. J Biol Chem. 2006 M603313200.

Ball JM, Hardy ME, Atmar RL, Conner ME, Estes MK. Oral immunization with recombinant Norwalk virus-like particles induces a systemic and mucosal immune response in mice. J Virol. 1998; 72:1345-1353. [PubMed: 9445035]

Chen Q. Expression and Purification of Pharmaceutical Proteins in Plants Biological Engineering. 2008; 1:291-321.

Chen, Q. Expression and manufacture of pharmaceutical proteins in genetically engineered horticultural plants. In: Mou, B.; Scorza, R., editors. Transgenic Horticultural Crops: Challenges and Opportunities - Essays by Experts. Taylor \& Francis; Boca Raton: 2011. p. 86-126.

Chen Q. Turning a new leaf. European Biopharm Rev. 2011; 2:64-68.

Chen, Q.; Tacket, CO.; Mason, H.; Mor, T.; Cardineau, GA.; Arntzen, C. Subunit vaccines produced using plant biotechnology. In: Levine, MM., editor. New Generation Vaccines. Informa Healthcare USA, Inc; New York: 2009. p. 306-315.

Davies HM. Commercialization of whole-plant systems for biomanufacturing of protein products: evolution and prospects. Plant Biotechnology Journal. 2010; 8:845-861. [PubMed: 20731788]

Elbehri A. Biopharming and the food system: examining the potential benefits and risks. AgBioForum. 2005; 8:18-25.

Estes, MK. Virus like particle (VLP) vaccines. In: Levine, MM., editor. New generation vaccines. Academic Press; New York: 2004. p. 283-294. 
Faye L, Gomord V. Success stories in molecular farming- a brief overview. Plant Biotechnology Journal. 2010; 8:525-528. [PubMed: 20500680]

Giritch A, Marillonnet S, Engler C, van Eldik G, Botterman J, Klimyuk V, Gleba Y. Rapid high-yield expression of full-size IgG antibodies in plants coinfected with noncompeting viral vectors. Proc Natl Acad Sci U S A. 2006; 103:14701-14706. [PubMed: 16973752]

He J, Lai H, brock C, Chen Q. A novel system for rapid and cost-effective production of detection and diagnostic reagents of West Nile virus in plants. J Biomed Biotech. 2011

Herbst-Kralovetz M, Mason HS, Chen Q. Norwalk virus-like particles as vaccines. Expert Rev Vaccines. 2010; 9:299-307. [PubMed: 20218858]

Huang Z, Chen Q, Hjelm B, Arntzen C, Mason H. A DNA replicon system for rapid high-level production of virus-like particles in plants. Biotechnol Bioeng. 2009; 103:706-714. [PubMed: 19309755]

Huang Z, Phoolcharoen W, Lai H, Piensook K, Cardineau G, Zeitlin L, Whaley K, Arntzen CJ, Mason $\mathrm{H}$, Chen Q. High-level rapid production of full-size monoclonal antibodies in plants by a singlevector DNA replicon system. Biotechnol Bioeng. 2010; 106:9-17. [PubMed: 20047189]

Jennings GT, Bachmann MF. Designing recombinant vaccines with viral properties: a rational approach to more effective vaccines. Curr Mol Med. 2007; 7:143-155. [PubMed: 17346167]

Jiang X, Wang M, Graham DY, Estes MK. Expression, self-assembly, and antigenicity of the Norwalk virus capsid protein. J Virol. 1992; 66:6527-6532. [PubMed: 1328679]

Komarova TV, Baschieri S, Donini M, Marusic C, Benvenuto E, Dorokhov YL. Transient expression systems for plant-derived biopharmaceuticals. Expert Review of Vaccines. 2010; 9:859-876. [PubMed: 20673010]

Lai H, Engle M, Fuchs A, Keller T, Johnson S, Gorlatov S, Diamond MS, Chen Q. Monoclonal antibody produced in plants efficiently treats West Nile virus infection in mice. Proc Natl Acad Sci U S A. 2010; 107:2419-2424. [PubMed: 20133644]

Lai H, He J, Engle M, Diamond MS, Chen Q. Robust production of virus-like particles and monoclonal antibodies with geminiviral replicon vectors in lettuce. Plant Biotechnol J. 2011

Lico C, Chen Q, Santi L. Viral vectors for production of recombinant proteins in plants. J Cell Physiol. 2008; 216:366-377. [PubMed: 18330886]

Patel MM, Widdowson MA, Glass RI, Akazawa K, Vinje J, Parashar UD. Systematic literature review of role of noroviruses in sporadic gastroenteritis. Emerg Infect Dis. 2008; 14:1224-1231. [PubMed: 18680645]

Paul M, Ma JKC. Plant-made immunogens and effective delivery strategies. Expert Review of Vaccines. 2010; 9:821-833. [PubMed: 20673007]

Prasad BV, Hardy ME, Dokland T, Bella J, Rossmann MG, Estes MK. X-ray crystallographic structure of the Norwalk virus capsid. Science. 1999; 286:287-290. [PubMed: 10514371]

Prasad BVV, Hardy D, Estes M. Structural Studies of Recombinant Norwalk Capsids. The Journal of Infectious Diseases. 2000; 181:S317-S321. [PubMed: 10804144]

Rolland D, Gauthier M, Dugua JM, Fournier C, Delpech L, Watelet B, Letourneur O, Arnaud M, Jolivet M. Purification of recombinant $\mathrm{HBc}$ antigen expressed in Escherichia coli and Pichia pastoris: comparison of size-exclusion chromatography and ultracentrifugation. Journal of Chromatography B: Biomedical Sciences and Applications. 2001; 753:51-65.

Rybicki E. Plant-made vaccines for humans and animals. Plant Biotechnology Journal. 2010; 8:620637. [PubMed: 20233333]

Santi L, Batchelor L, Huang Z, Hjelm B, Kilbourne J, Arntzen CJ, Chen Q, Mason HS. An efficient plant viral expression system generating orally immunogenic Norwalk virus-like particles. Vaccine. 2008; 26:1846-1854. [PubMed: 18325641]

Speicher, KD.; Gorman, N.; Speicher, DW. Current Protocols in Protein Science. John Wiley \& Sons, Inc; 2001. N-Terminal Sequence Analysis of Proteins and Peptides.

Thiel KA. Biomanufacturing, from bust to boom...to bubble? Nature biotechnology. 2004; 22:13651372.

Velasquez LS, Hjelm BE, Arntzen CJ, Herbst-Kralovetz MM. An intranasally delivered Toll-like receptor 7 agonist elicits robust systemic and mucosal responses to Norwalk virus-like particles. Clin Vaccine Immunol. 2011; 17:1850-1858. [PubMed: 20962211] 
Zhang X, Buehner NA, Hutson AM, Estes MK, Mason HS. Tomato is a highly effective vehicle for expression and oral immunization with Norwalk virus capsid protein. Plant Biotechnol J. 2006; 4:419-432. [PubMed: 17177807] 
Key Message : Successful first demonstration of scale-up production of pharmaceutical grade Norwalk virus capsid protein vaccine in plants under current Good Manufacture Practice regulations and the quality of this vaccine meets regulatory standards in identity, purity, and potency. 

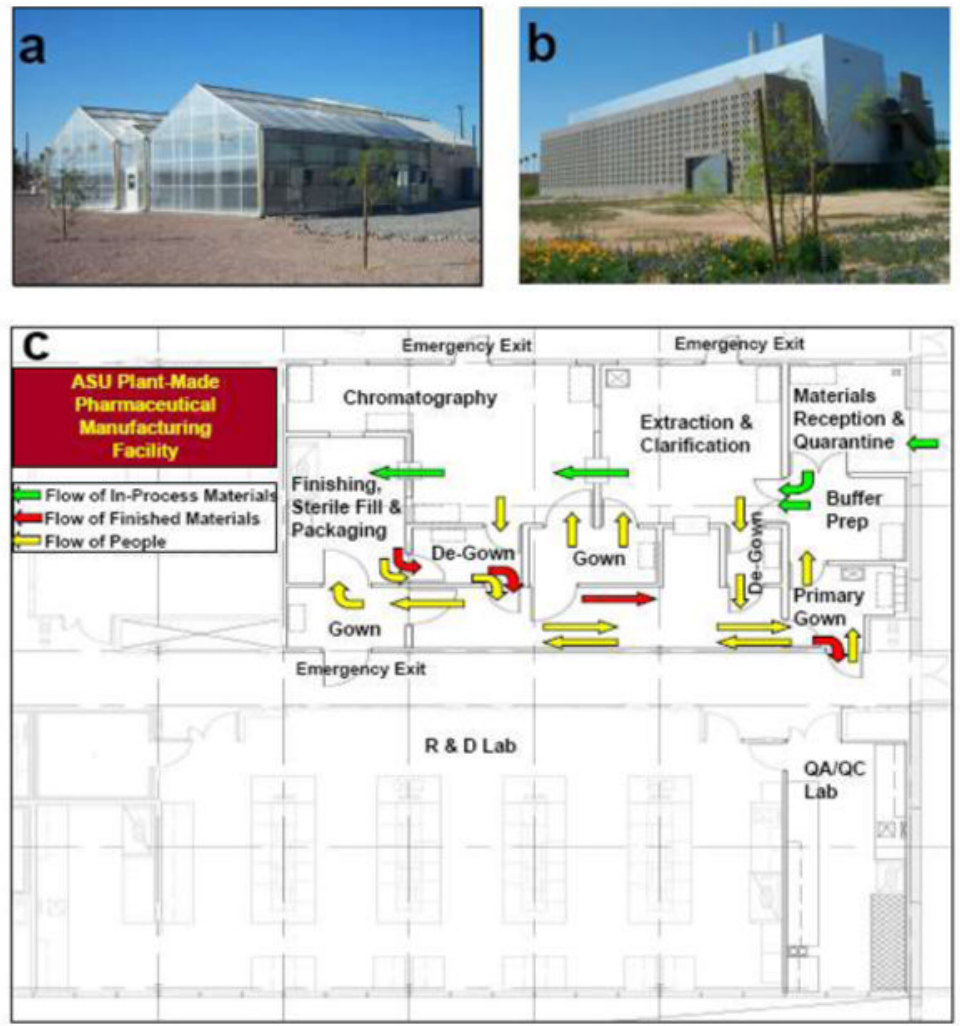

Fig. 1.

Facilities for cGMP production of NVCP VLP vaccine and other plant-made pharmaceuticals. (a) The $3600 \mathrm{ft}^{2}$ BSL-2 greenhouse facility for plant biomass generation and NVCP expression. (b) The Plant Biopharmaceutical Center that houses the Central Bioprocessing Suite, the QA/QC laboratory, and the Process Development laboratory. (c) The floor plan of the Central Bioprocessing Suite. The arrows indicate the unidirectional flow of in-process materials (green), purified final product (red) and people (yellow). 

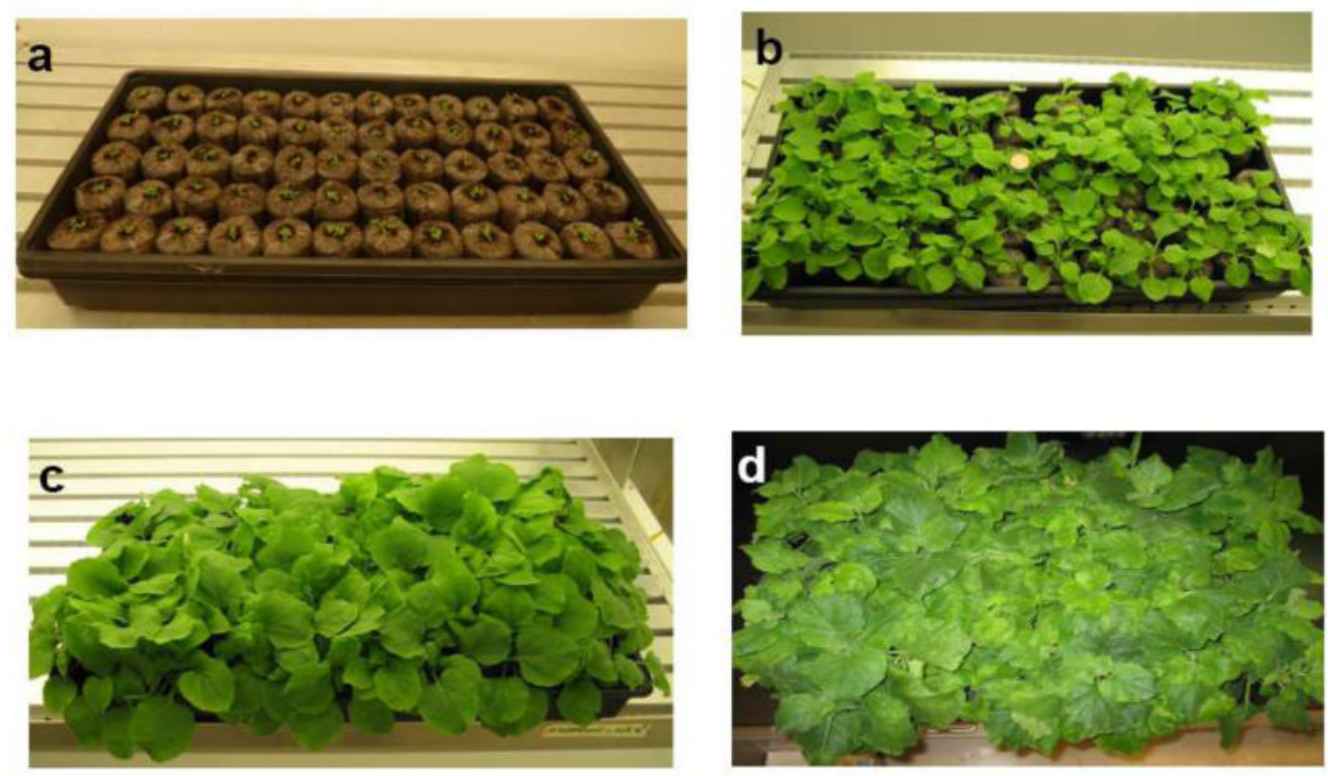

Fig. 2.

Biomass generation with Jiffy-7 peat pellets and growth trays under production conditions optimized in this study. Wild-type $N$. benthamiana plants one (a), three (b), four (c), and five (d) weeks after seed sowing. 


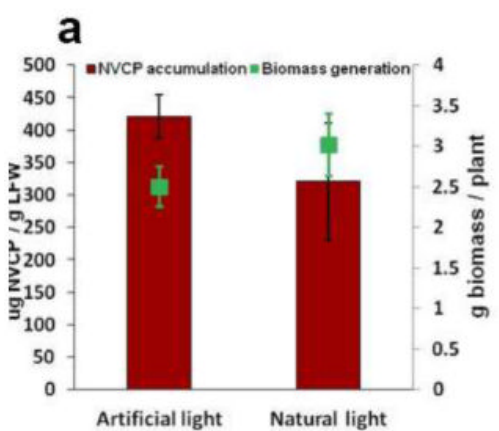

C

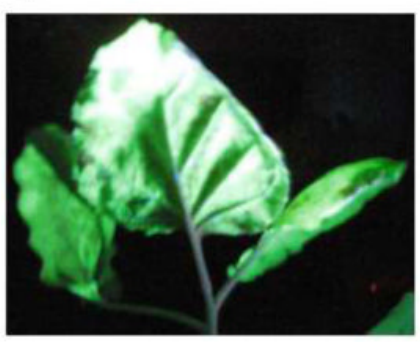

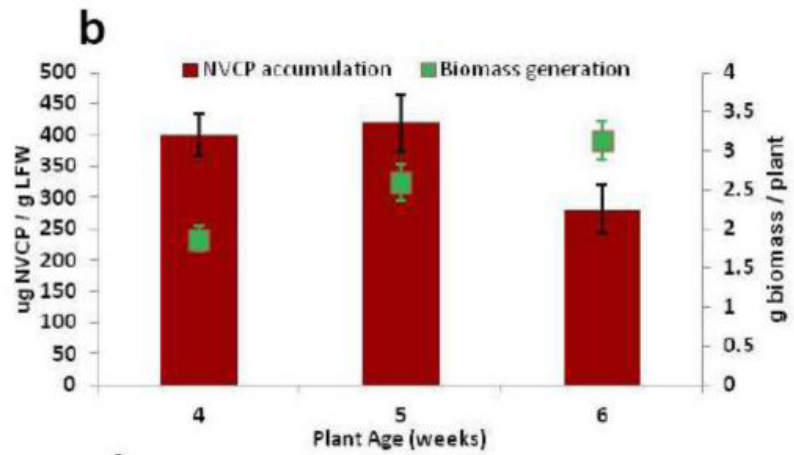

d

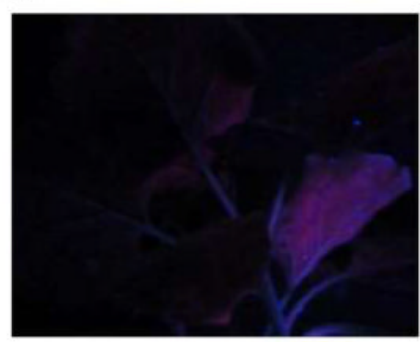

Fig. 3.

Optimization of biomass generation and NVCP expression. (a) Biomass and NVCP yield under natural and artificial light. $N$. benthamiana plants were growth either under natural or artificial light for 5 weeks. Leaf biomass (green square) and NVCP expression level (red column) were measured by weighing and ELISA, respectively. (b) Temporal pattern of biomass yield and NVCP expression. Plants were grown under artificial light for 5 weeks, and biomass production (green square) and NVCP accumulation (red column) were examined. For both (a) and (b), mean \pm standard deviation (SD) of samples (N>10) from three independent infiltration experiments are presented. (c) and (d) Visualization of GFP expression in Agroinfiltrated leaves. $N$. benthamiana plants were infiltrated either with GV3101 cultures that carrying the three MagnICON vectors for GFP expression (c) or with infiltration buffer as a negative control (d). Leaves were examined and photographed 7 dpi under UV light. One representative of at least three independent experiments is shown. 


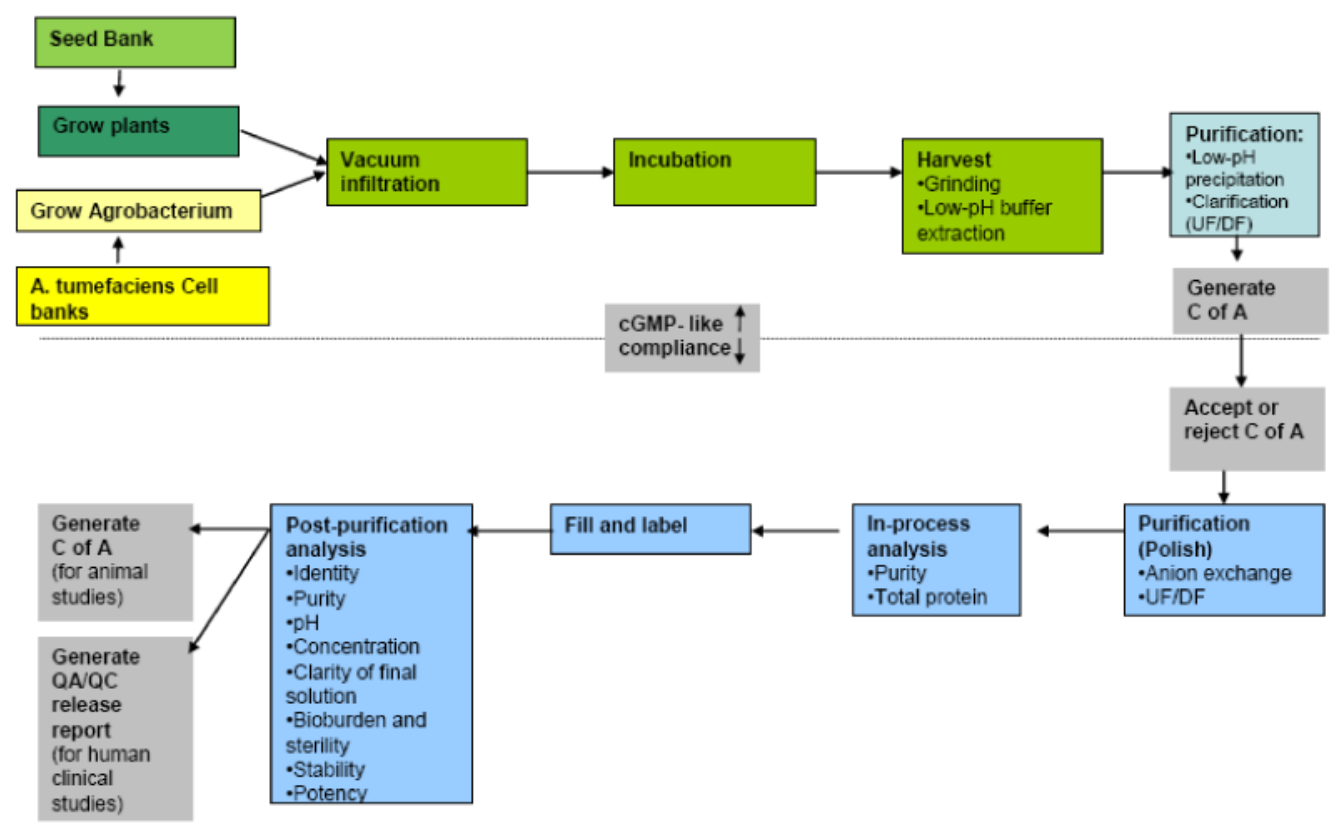

Fig. 4.

Process flow for the production of NVCP VLP vaccine under cGMP regulations. C of A: certificate of analysis, UF/DF: ultrafiltration and diafiltration. 


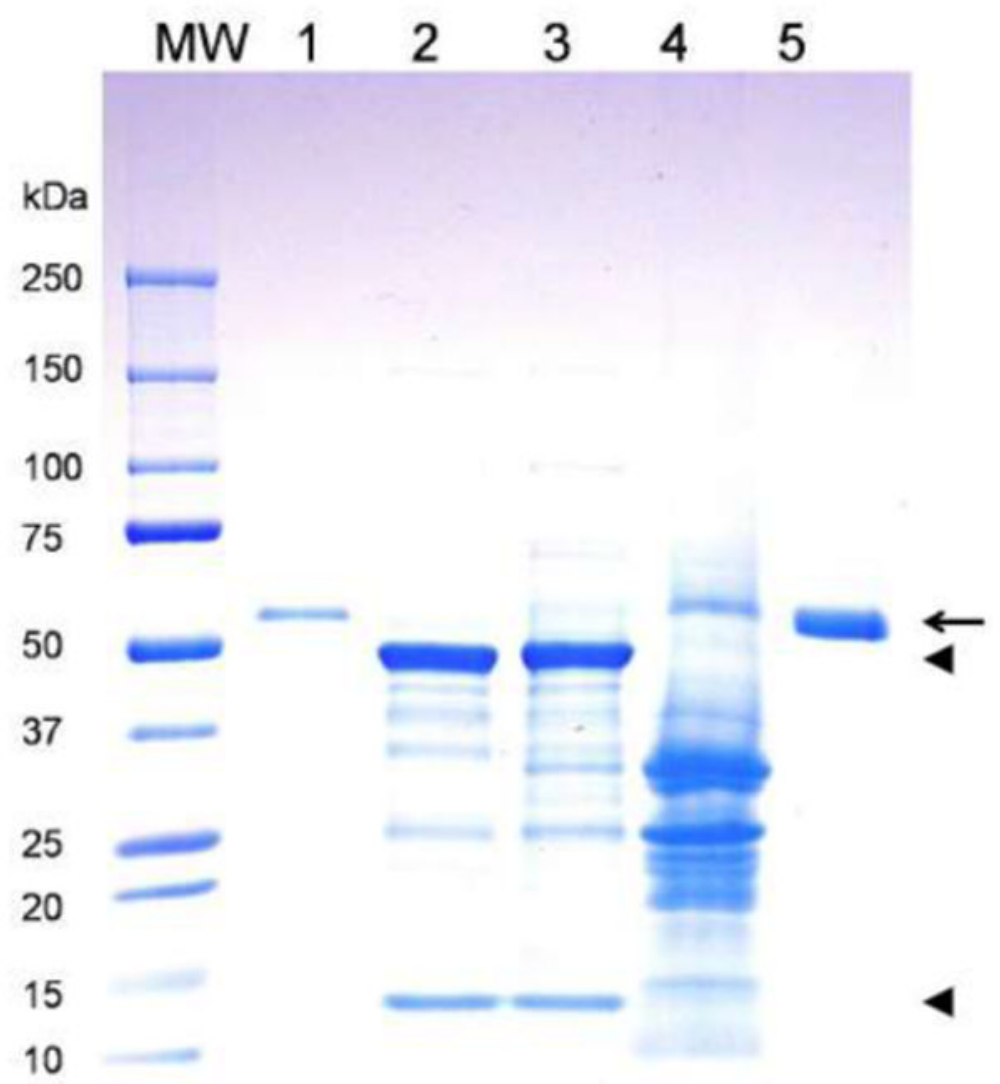

Fig. 5.

Purification of NVCP from $N$. benthamiana plants. Leaf protein extract was purified and analyzed on a 4-20\% SDS-PAGE gel under reducing condition. Lane 1, Insect cell-produced NVCP as a reference standard; Lane 2, Clarified leaf extract from uninfiltrated plants; Lane 3, Clarified leaf extract from NVCP-producing plants; Lane 4, Supernatant of low-pH precipitation; Lane 5, Purified plant-derived NVCP from DEAE anion-exchange chromatography. 4 : RuBisCo large and small subunits; $\nleftarrow:$ NVCP. 
Table 1

Scalability of plant-derived NVCP VLP downstream processing

\begin{tabular}{|ccc|}
\hline LFW(g) & Overall recovery (\%) & Purity \\
\hline 100 & $33.08 \pm 6.55$ & $>95 \%$ \\
500 & $25.41 \pm 7.38$ & $>95 \%$ \\
15,000 & $23.76 \pm 9.47$ & $>95 \%$ \\
\hline
\end{tabular}


Table 2

\section{Analytical assays for NVCP VLP in-process samples and the final purified product}

\begin{tabular}{|cc|}
\hline Product Attribute & Analytical Method \\
\hline Product identity & Western blot \\
Product identity & N-terminal sequence with Automated Edman sequencing \\
Purity and molecular & SDS-PAGE/profile Coomassie and Silver staining \\
Protein purity and concentration & RP-HPLC \\
Protein concentration & Absorption at wavelength of 280nm (A280) \\
Protein concentration & Amino Acid Analysis (AAA) \\
Mass & ESI-TOF mass spectrometry (ESI-TOF MS) \\
Quaternary structure & SEC HPLC Multi-angle laser light scattering (MALLS) \\
Quaternary structure & Sucrose gradient centrifugation, TEM \\
Determination of pI & Isoelectric focusing (IEF)-PAGE \\
Glycan analysis & MALDI-TOF MS \\
Endotoxin & Limulus amebocyte lysate \\
Bioburden & Turbidity \& colony formation \\
Residual host DNA & PicoGreen and PCR \\
Small molecules, Nicotine & LC-MS/MS \\
Residual host cell protein & Polyclonal Abs to wild-type $N$. Benthamiana plant protein \\
\hline
\end{tabular}

SDS-PAGE: sodium dodecyl sulphate polyacrylamide gel electrophoresis, RP-HPLC: reverse phase high pressure liquid chromatography, ESITOF: electrospray ionization-time of flight, TEM: transmission electron microscopy, MALDI: matrix-assisted laser desorption-ionization, PCR: polymerase chain reaction, LC-MS/MS: liquid chromatography-tandem mass spectrometry. 
Table 3

\section{Characteristics of the final purified NVCP VLP product}

\begin{tabular}{|ccc|}
\hline Product Attribute & Result and Assay & $\begin{array}{c}\text { Comparison with } \\
\text { iNVCP standard }\end{array}$ \\
\hline Identity & The first 6 Amino acids at the $\mathrm{N}$-terminus have the same sequence as the iNVCP & conform \\
standard & Single $58 \mathrm{kDa}$ band on western blot & conform \\
Molecular size and identity & $>95 \%$ pure, single $58 \mathrm{kDa}$ band on SDS-PAGE, & conform \\
Purity and molecular size & Colorless liquid, slightly cloudy, by visual observation & conform \\
Appearance & $10 \pm 0.3 \mathrm{mg} / \mathrm{ml}$ by A280 & conform \\
Protein concentration & $5.75 \pm 0.03$ & conform \\
$\mathrm{pH}$ & $38 \mathrm{~nm}$ VLP by TEM microscopy & conform \\
Quaternary structure & conform \\
Quaternary structure & Detected as VLP by sucrose gradient centrifugation & conform \\
Residual host DNA & < $1 \mathrm{ng} / \mathrm{ml}$ by PicoGreen Assay & conform \\
Product stability & Stable $>2$ years at $-80 \pm 4{ }^{\circ} \mathrm{C}$ by SDS-PAGE and Western Blot & conform \\
Product potency & Serum NV-specific IgG and mucosal NV-specific IgA production in mice following & intranasal immunization (Velasquez, et al. 2011) \\
\hline
\end{tabular}

iNVCP: insect cell-derived NVCP VLP standard. 